\title{
KRONECKER SETS AND METRIC PROPERTIES OF $M_{0}$-SETS
}

\author{
ROBERT KAUFMAN
}

\begin{abstract}
A method for constructing both sets of multiplicity and Kronecker sets within a given set of multiplicity is derived from the work of Ivashev-Musatov; it is shown that the Hausdorff measures and other measures are essentially distinct. Finally, an improvement of a theorem of Salem is obtained, using PyateckiiShapiro's theorem on non- $M$ sets.
\end{abstract}

1. The class of complex Borel measures $\mu$ on the real axis, such that $\hat{u}(u) \rightarrow 0$ as $|u| \rightarrow \infty$, is denoted $R$, and a closed set is called $M_{0}$ if it supports some measure $\mu \neq 0$ of class $R$. With a measure $\mu$ the entire space $L^{1}(\mu)$ is contained in $R$ [7, I, p. 143], so that we mostly study probability measures in $R$. The most striking examples of non- $M_{0}$ sets are the Kronecker sets: A compact linear set $E$ is a $K$-set if each continuous function on $E$ of modulus 1 admits uniform approximation on $E$ by characters $\%(x)=$ $e^{i u x}(-\infty<u<\infty)([2],[6])$.

An easy application of Fubini's theorem shows that if $E_{1}$ and $E_{2}$ are closed, $E_{1}$ is $M_{0}$ and $m\left(E_{2}\right)>0$, then $E_{1} \cap\left(E_{2}+x\right)$ is $M_{0}$ for an $x$-set of positive Lebesgue measure. A sharp converse is true.

THEOREM 1. Let $F$ be a closed set of Lebesgue measure $m(F)=0$. Then there exists an $M_{0}$-set $E$ so that $(F+x) \cap E$ is a $K$-set for every real $x$.

Theorem 1 is a consequence of a more general assertion; for closed sets $F$ and numbers $r>0$ let $m(F, r)=m\{x: \operatorname{dist}(x, F) \leqq r\}$.

THEOREM 1'. Let $g$ be any positive, increasing function on $(0,1)$ and let $g(0+)=0$. Then there is an $M_{0}$-set $E$ such that each closed set $F \subseteq E$ is a $K$-set, unless $m(F, r)>g(r)$ for all $r \leqq r_{0}(F)$.

Theorem $1^{\prime}$ is derived from a very general theorem in which Lebesgue measure plays no special role.

THEOREM 2. Let $\mu$ be a probability of class $R$ and of closed support $S$; let $\left(T_{k}\right)_{1}^{\infty}$ be a sequence of closed sets with $\lim \mu\left(T_{k}\right)=0$. Then there exists an

Received by the editors January 19, 1972.

AMS (MOS) subject classifications (1969). Primary 4250, 4252; Secondary 4256.

Key words and phrases. $K$-set, $M_{0}$-set, $M$-set, uniform distribution, Diophantine approximation, Fourier transform.

(c) American Mathematical Society 1973 
$M_{0}$-set $S_{0} \subseteq S$ with this property: for every infinite set I of integers $k, S_{0} \cap$ $\bigcap_{I} T_{k}$ is a K-set.

The proof of Theorem 2 requires a few preliminary remarks and adjustments.

(a) There are open sets $W_{k} \supseteq T_{k}$ whose boundaries $\partial W_{k}$ have $\mu$-measure 0 , and moreover $\mu\left(W_{k}\right)<\mu\left(T_{k}\right)+k^{-1}$. This much is true for Baire measures in any metric space. In the set of probability measures in $L^{1}(\mu)$, the mappings $\lambda \rightarrow \lambda\left(W_{k}\right)$ are ${ }^{*}$-continuous, because $\mu\left(\partial W_{k}\right)=0$. In the same direction, let $V$ be an open set; the set of probabilities such that $\lambda(V)=0$ is $w^{*}$-closed.

(b) A distance function on the Borel measures is defined by the formula $p\left(\lambda_{1}, \lambda_{2}\right)=\sup \left|\lambda_{1}\left(W_{k}\right)-\lambda_{2}\left(W_{k}\right)\right|$. In the proof we shall construct probability measures $\mu_{j}$ in $L^{1}(\mu)$ such that $\mu=\mu_{0}$ and

(i) $p\left(\mu_{j}, \mu_{j+1}\right) \leqq 2^{-j}$ for $j=0,1,2, \cdots$.

(ii) $\left|\hat{\mu}_{j}(u)-\hat{\mu}_{j+1}(u)\right| \leqq 2^{-j}$ for every $u$, with at most one exception $j=j(u)$.

(iii) $\left\|\mu_{j+1}-\mu_{j}\right\| \leqq 2 \mu_{j}\left(W_{j}\right)$.

From (i) it will follow that $\lim \mu_{j}\left(W_{j}\right)=0$ and then from (ii) and (iii) that $\hat{\mu}_{j}$ converges uniformly on the real axis, to a function also tending to 0 at infinity. The property $\mu\left(\partial W_{k}\right)=0$ is used for (i).

(c) Let $\lambda$ be a probability measure of class $R$, and $f$ a real measurable function. Then the measurable functions of $x, u x-f(x)$, tend to uniform distribution (modulo 1) in $\lambda$-measure, as $|u| \rightarrow \infty$. To prove this we use Weyl's criterion [1, IV] for uniform distribution, with the notation $e(t) \equiv \exp 2 \pi i t$. It must be proved that

$$
\lim \int e(k u x-k f(x)) \lambda(d x)=0 \text { for } k=0,1,2, \cdots
$$

But the complex measures $e(-k f(x)) \lambda(d x)$ are of class $R$ and this fact expresses the limit relation required.

(d) Let now $S$ be compact and totally disconnected, as is easily achieved, and $\left(g_{k}\right)_{1}^{\infty}$ be a dense sequence in the real Banach space $C(S)$. We shall use the fact that, on $S$ and any closed subset of $S$, a continuous function of modulus 1 admits uniform approximation by the subset $e\left(g_{k}\right)$. We construct closed sets $A_{j} \subseteq W_{j}$ and measures $\mu_{j}$ by the formulas

$$
\mu_{j+1}\left(B \sim W_{j}\right) \equiv \mu_{j}\left(B \sim W_{j}\right), \quad \mu_{j+1}\left(B \cap W_{j}\right) \equiv c_{j} \mu_{j}\left(B \cap A_{j}\right)
$$

for appropriate constants $c_{j}>0$, so that $\mu_{j+1}$ is again a probability. As $\mu_{j+1}$ and $\mu_{j}$ differ only by a certain adjustment outside of $W_{j}$, an easy calculation yields (iii). Moreover, to each function $g_{1}, \cdots, g_{j}$ there will be integers $u_{1}, \cdots, u_{j}$ so that $\left|u_{1}(x)-g_{1}(x)\right|<2^{-j}$ (modulo 1 ), $\left|u_{j}(x)-g_{j}(x)\right|<$ $2^{-j}$ (modulo 1), on $A_{j}$. Observe also that the measures $\mu_{j}$ have decreasing 
closed supports; this, with the last remark in (a), shows that any $w^{*}$ accumulation point $\lambda$ of the sequence $\left(\mu_{j}\right)$ has support $S_{0}$ such that $S_{0} \cap T_{j} \subseteq S_{0} \cap W_{j} \subseteq A_{j}$ so that $S_{0}$ has the 'Kronecker' properties asserted.

The measures $\mu_{j}$ are constructed by induction; suppose this accomplished up to $\mu_{s}$. Because $\mu_{0}, \cdots, \mu_{s}$ are of class $R$ (belonging to $L^{1}(\mu)$ ), there is a number $Y$ such that $\left|\hat{\mu}_{j}(u)\right|<2^{-s-1}$ for $0 \leqq j \leqq s$ whenever $|u| \geqq Y$. Thus requirement (ii) need only be attained on the interval $|u| \leqq Y$.

(e) Let $0<\delta<2^{-s} Y^{-1}$, and let $\left(W_{n}\right)$ be a finite partition of $W_{s}$ into subsets of diameter $<\delta$. When the measure $\mu_{s+1}$ has the property that $\mu_{s+1}\left(W_{n}\right)=\mu_{s}\left(W_{n}\right)$ for each, $n$, then $\left|\hat{\mu}_{s+1}(u)-\hat{\mu}_{s}(u)\right| \leqq \delta|u|<2^{-s}$ on $[-Y, Y]$. It remains to construct the set $A_{s}$ and attain inequality (i).

Using Weyl's criterion $s$ times in succession, we find that there are integers $u_{1}, \cdots, u_{s}$ so that the set $\left\{\left|u_{j} x-g_{j}(x)\right|<2^{-s}\right.$ (modulo 1), $1 \leqq j \leqq s\}$ meets each $W_{n}$ in a set of $\mu_{s}$-measure at least $2^{-s-1} \mu_{s}\left(W_{n}\right)=$ $a_{s} \mu_{s}\left(W_{n}\right)$. Here $a_{s}$ depends only on $s$. Then, using the regularity of Borel measures, there is a closed set $A_{s}$ on which the required approximations are valid, while $\mu_{s}\left(W_{n} \cap A_{s}\right)={ }_{2}^{1} a_{s} \mu_{s}\left(W_{n}\right)$. Thus $\mu_{s+1}$ can be defined with $c_{s}=2 a_{s}^{-1}$ (or, in fact, any number $c_{s}>2^{s}$ ).

(f) To obtain inequality (i) we note that all the measures $\mu_{s+1}$ arising in this construction satisfy the same inequality $0 \leqq \mu_{s+1} \leqq c_{s_{s}} \mu_{s}$ (as set functions), whence $\mu_{s+1}\left(W_{k}\right) \leqq c_{s} \mu_{s}\left(W_{k}\right)$ for all measures $\mu_{s+1}$. Thus the inequality $\left|\mu_{s+1}\left(W_{k}\right)-\mu_{s}\left(W_{k}\right)\right| \leqq 2^{-s}$ is a constraint only when $c_{s} \mu_{s}\left(W_{k}\right)>$ $2^{-s-1}$, that is for $k \leqq k_{s}$, say. For these values of $k$, we use the $w^{*}$-continuity of the mappings $\left.\lambda_{i} \rightarrow \lambda_{k}\right)$ on $L^{1}(\mu)$, by making $\delta>0$ small enough to bring each $\mu_{s+1}$ close to $\mu_{s}$ in the $w^{*}$-topology. This completes the construction of $\mu_{s+1}$ and the proof of Theorem 2. It is worthwhile remarking that if any sequence $e\left(u_{m} . x\right)$ of characters is assigned in advance, with $\left|u_{m}\right| \rightarrow \infty$, then the Kronecker property of the intersections $S_{0} \cap \bigcap_{I} T_{k}$ can be enforced using only these characters.

Proof of THEOREM $1^{\prime}$. Let $S$ be an interval of length 1 and $\mu$ the Lebesgue measure on $S$. Let $J(n)$ be the usual division of $S$ into intervals of length $n^{-1}, C(n)$ the set of unions of at most $n g\left(2 n^{-1}\right)$ of these intervals, and finally let $\left(T_{k}\right)_{1}^{\infty}$ be an enumeration of $C(n)$. To see that Theorem 2 applied with the present choice of $\mu$ and $\left(T_{k}\right)$ yields Theorem $1^{\prime}$, consider a subset $F \subseteq S$ such that $m(F, r) \leqq g(r)$ for some $r$ in $(0,1)$. Defining $n$ by the inequalities $n^{-1}<r \leqq(n-1)^{-1}$, we see that $F$ meets at most $n m(F, r) \leqq$ $n g(r) \leqq n g\left(2 n^{-1}\right)$ intervals from $J(n)$, and Theorem $\mathrm{l}^{\prime}$ is proved.

To see the special properties of the measure function $m(F, r)$, we compare it with Hausdorff measure [5, II]. Recall that if $h$ is increasing and positive on $(0, \infty)$ and $h(0+)=0$, a set $F$ has $h$-measure 0 if, to each

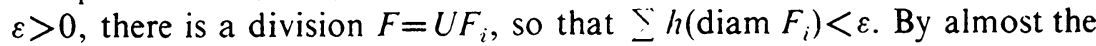
same arguments as before, the following improvement of the theorem of Ivashev-Musatov is obtained [3]. 
THEOREM 3. Each $M_{0}$-set E contains an $M_{0}$-set $E_{1}$ of h-measure 0 .

2. Theorems $1^{\prime}$ and 3 are in obvious contrast, and another contrast to Theorem 3 is obtained from Dirichlet's theorem on simultaneous approximation ([1, I]; [7, II, p. 153]).

THEOREM 4. Let $\mu$ be a probability of class $R$ and $F_{k}$ any union of $C \log k$ intervals of length $k^{-1}$. Then $\lim \mu\left(F_{k}\right)=0$.

Proof. We shall first show that $\lim \sup \mu\left(F_{k}\right) \leqq 2 \exp -C^{-1}$. In fact let $\exp -C^{-1}<b<1$ and $\delta>0$, and let $x_{1}, \cdots, x_{x}(N \leqq C \log k)$ be the centers of the given intervals. The inequalities

$$
\left.\left|\xi x_{j}\right| \leqq b \text { (modulo } 1\right), \quad 1 \leqq j \leqq N,|\xi| \leqq \delta k,
$$

have an integer solution $\xi>0$ as soon as $N \log b+\log \delta+\log k>0$, and in fact the largest solution, say $\xi_{k}$, tends to $+\infty$ because $C \log b>-1$. (See the remarks below on Dirichlet's theorem.) Because $1 \leqq \xi_{k} \leqq \delta k$ we find $\left|\xi_{k} x\right| \leqq b+\delta$ (modulo 1) for every $x$ in $F_{k}$ and by Weyl's criterion, $\lim \sup \mu\left(F_{k}\right) \leqq 2(b+\delta)$.

Next, for any integer $r \geqq 1$, the intervals comprised in $F_{k}$ can be divided into $r$ approximately equal classes; this yields the estimate

$$
\lim \sup \mu\left(F_{k}\right) \leqq 2 r \exp -r C^{-1},
$$

whence $\lim \mu\left(F_{k}\right)=0$.

This theorem is to be compared with Theorem 3, for it gives a sort of modulus of continuity. In particular, $F$ is non- $M_{0}$ provided $\lim \inf m(F, r) / r \log r^{-1}<\infty$. This result is very sharp, as shown by a theorem of Kahane [4]. (Theorem 4 resolves the question raised at the conclusion of [4].)

In both Theorem 4 and the following argument, it is necessary to obtain a large number of solutions in Dirichlet's theorem on simultaneous approximation. The references cited deduce Dirichlet's theorem from Minkowski's theorem on lattice points in a symmetric convex set $S$ in the Euclidean space $R^{p}$. However, if $2^{-p} V(S)$ is large, the analysis of Minkowski's theorem yields a large number of lattice points, and these determine distinct solutions in the Dirichlet theorem.

Besides the concept of $M_{0}$-set, there is also that of $M$-set, defined as follows (for closed sets, at any rate). A closed set $E$ is an $M$-set if it supports a distribution $T \neq 0$ whose Fourier transform $\hat{T}$ belongs to $L^{\infty}$ and vanishes at infinity. This is plainly not the language of Riemann and Cantor; a more traditional definition is adopted in [7], and the equivalence of the two theories is explained in [5, V]. The following theorem describes a stronger property of a closed set than that deduced from Theorem 4, 
but does not contain Theorem 4, because it does not concern measures at all.

THEOREM 5. Let $F$ be a closed set contained in $C \log p_{k}^{-1}$ intervals of length $p_{k}$ for a certain sequence $p_{k} \rightarrow 0$. Then $F$ is not an $M$-set.

The proof requires knowledge of the work of Pyateckir-Shapiro [7, I, p. 346], especially the definition of an $H^{(r)}$-set. To obtain this theorem, we observe that $F$ is contained in sets of type $F_{k}$, for a sequence of integers $k$ tending to infinity.

Given the constant $C>0$, let $r>C \log 2$ be an integer, and $0<b<\frac{1}{2}$ while $\log b>-r C^{-1}$. The integers $j$ in the interval $[1, C \log k]$ are divided into $r$ nearly equal classes, say $L_{1}, \cdots, L_{r}$. The Diophantine inequalities, for $n=1, \cdots, r$,

(I $\left.{ }_{n}\right) \quad\left|\xi_{n} x_{j}\right| \leqq b($ modulo 1$)$ for $j$ in $L_{n}, \quad\left|\xi_{n}\right| \leqq \frac{1}{4}(1-2 b) k \equiv \delta k$,

have solutions $\xi_{n}$ forming a set $Q_{n}$, whose cardinalities tend to infinity. Here of course the inequality $-\log b<r C^{-1}$ is decisive. Compare [5, p. 95].

To apply Pyateckiĭ-Shapiro's method $[7, \mathrm{I}, \mathrm{p} .346]$ we must choose special $r$-tuples $\left(\xi_{1}, \cdots, \xi_{r}\right)$ from $Q=Q_{1} \times \cdots \times Q_{r}$. More exactly, given any finite union $U$ of linear inequalities of the form

$$
\left|a_{1} \xi_{1}+\cdots+a_{r} \xi_{r}\right| \leqq A, \quad \max \left|a_{n}\right| \geqq 1,
$$

we must choose a point $\left(\xi_{1}, \cdots, \xi_{r}\right)$ in $Q \sim U$. Now in one of our inequalities, with (say) $\left|a_{r}\right| \geqq 1$, any $\xi_{1}, \cdots, \xi_{r-1}$ admits at most $2 A+1$ choices for $\xi_{r}$, so $U$ meets $Q$ in a very small part, that is $|Q \cap U|=o(|Q|)$ so that $Q \sim U$ is nonvoid for large $k$.

Consequently, a set $F$ is of type $H^{(r)}$ provided it is contained in $C \log k$ intervals of length $k^{-1}$, for an unbounded sequence of integers $k$. This conclusion, with $C \log k$ strengthened to $o(\log k)$, is due to Salem [5, p. 95]; [4] and [5] treat slightly different properties, and for these $o(\log k)$ is the critical case.

\section{REFERENCES}

1. J. W. S. Cassels, An introduction to Diophantine approximation, Cambridge Tracts in Math. and Math. Phys., no. 45, Cambridge Univ. Press, New York, 1957. MR 19, 396.

2. E. Hewitt and S. Kakutani, $A$ class of multiplicative linear functionals on the measure algebra of a locally compact Abelian group, Illinois J. Math. 4 (1960), 553-574. MR 23 \#A527.

3. O. S. Ivašev-Musatov, M-sets and h-measures, Mat. Zametki 3 (1968), 441-447. (Russian) MR 37 \#3273. 
4. J.-P. Kahane, A metric condition for a closed circular set to be a set of uniqueness, J. Approximation Theory 2 (1969), 233-236. MR 40 \#631.

5. J.-P. Kahane and R. Salem, Ensembles parfaits et séries trigonométriques, Actualités Sci. Indust., no. 1301, Hermann, Paris, 1963. MR 28 \#3279.

6. W. Rudin, Fourier analysis on groups, Interscience Tracts in Pure and Appl. Math., no. 12, Interscience, New York, 1962. MR 27 \#2808.

7. A. Zygmund, Trigometrical series, 2nd rev. ed., Cambridge Univ. Press, New York, 1959. MR 21 \#6498.

Department of Mathematics, University of Illinois, Urbana, Illinois 61801 